\title{
Hematologic Improvement-Neutrophil Response
}

National Cancer Institute

\section{Source}

National Cancer Institute. Hematologic Improvement-Neutrophil Response. NCI

Thesaurus. Code C123586.

An improvement of neutrophil count as a response to treatment. 